\title{
CUANTIFICACIÓN DE LOS RECURSOS EMPLEADOS POR EL SECTOR PÚBLICO Y PRIVADO PARA MITIGAR LOS DAÑOS OCASIONADOS POR LOS INCENDIOS FORESTALES EN EL MUNICIPIO DE IBAGUÉ TOLIMA COLOMBIA. \\ QUANTIFICATION OF THE RESOURCES USED BY THE PUBLIC AND PRIVATE SECTOR TO MITIGATE THE DAMAGED CAUSED BY FOREST FIRES IN THE CITY OF IBAGUÉ TOLIMA COLOMBIA.
}

Recebimento: 06/05/2017- Aceite: 15/08/2017- Publicação: 23/12/2017

Processo de Avaliação: Double Blind Review

Mario Heimer Flórez Guzmán ${ }^{1}$

Ph.D en Ciencias de la educación

Investigador de Uniremington

Investigador Grupo de Investigación PLANAUDI

mariohfg@hotmail.com

Luisa Fernanda Forero Herrera

Estudiante de la Especialización en Revisoría Fiscal y Contadora Público

herrera-luisa.foreroh@gmail.com

Luz Amparo Cardona Henao

Diplomado en Normas Internacionales para PYMES, estudiante de la Especialización en Revisoría Fiscal y Contador Público

ach215@hotmail.com

Oscar Javier Leytón Varón

Diplomado en Normas Internacionales para PYMES, Estudiante de la especialización en Revisoría Fiscal y Contador Público, Administrador Financiero,

oleytonvaron_contabilidad@yahoo.com

\section{RESUMEN}

El presente artículo de investigación de corte descriptivo, da cuenta de la cantidad de recursos que ha empleado el sector público y privado para la mitigación de los impactos que en materia ambiental y social han generado los incendios forestales desencadenados en la ciudad de Ibagué que pertenece al departamento del Tolima en Colombia. Dentro del constructo

${ }^{1}$ Autor para correspondência: Pioneer Plaza, 900 Fort Street Mail 410, Honolulu, HI 96813, Estados Unidos. 
metodológico la investigación es de corte descriptiva, en donde las variables a estudiar por los autores fueron: las causas, consecuencias de los incendios forestales y los costos asumidos por la administración pública, así como por entes privados para la mitigación de los impactos que en materia social y ambiental se han generado por causa de estos incendios. Como conclusión se obtuvo que la mayoría de incendios forestales en la ciudad de Ibagué fueron acusados por situaciones antrópicas, lo cual denota falta de conciencia ambiental por parte de los pobladores o visitantes de las localidades afectadas, así como de que la relación entre daño ocasionado y acción correctiva, no es suficiente para mitigar específicamente los daños ocasionados, especialmente en términos de flora y fauna.

PALABRAS CLAVE: Incendios Forestales, Reforestación, Sostenibilidad, Impactos Sociales y Ambientales

\section{ABSTRACT}

The present paper displays the quantity of resources that have been utilized by the public and private sector in order to mitigate the impacts in terms of social and environmental facts wich have been generated for forest fires in the Ibagué Tolima Colombia. Within the metodological construct it's a descriptive research, where the variables object of study determined by the authors are: causes, consequences of the forest fires and the assumed costs for the public administration, as well as private entities in order to mitigate the impacts in terms of social and enviromental facts generated for those forest fires. As conclusion, was identified that most of the forest fires in the city of Ibague where accused by anthropic situations, which can be inferred that there is a weak in term of inveromental conscious in people living and visiting the affected areas, as well as the relation between danage caused and corrective actions, is not enough to mitigate specifically the damage caused, especially in terms of flora and fauna.

KEY WORDS: Forest Fires, Reforestation, Sustainability, Social and Environmental Impacts

\section{INTRODUCCIÓN}

Este artículo mostrará una estimación del impacto económico provocado por los incendios forestales en el municipio de Ibagué - Tolima en el año 2015, elaborado por un grupo de estudiantes de la Especialización en Revisoría Fiscal y Auditoría Integral de la Universidad Cooperativa de Colombia, sede Ibagué.

Con el propósito de evaluar a fondo la problemática ambiental que golpeó fuertemente al Municipio de Ibagué - Tolima durante el año 2015, donde el fenómeno cálido del Pacífico (Fenómeno del "Niño") pasó, dejando grandes pérdidas en biodiversidad, lo cual representó un quebranto funcional y estructural de los ecosistemas y una afectación grave a

Revista ENIAC Pesquisa, Guarulhos (SP), V.6, n.2, jun.- dez. 2017 
la sociedad. Para su estudio se realiza un proceso de auditoría ambiental - ecológica, tomando información de distintas fuentes documentales; donde el enfoque principal será conocer el costo en que deberá incurrir el estado para el restablecimiento de los daños ocasionados por los incendios forestales.

El Municipio de Ibagué es un punto estratégico ${ }^{2}$ de la geografía nacional de Colombia, debido a su ubicación -centro-occidente- de Colombia sobre la Cordillera Central de los Andes; Los incendios forestales han provocado la carencia de agua en áreas que antes se caracterizaban por su abundancia, la pérdida de cobertura vegetal nativa, impactando negativamente el bienestar y la calidad de vida de la Población, limitando sus posibilidades de desarrollo y comprometiendo gravemente las generaciones futuras en cuanto a la sostenibilidad de su desarrollo (Freeman, 2015).

\section{REFERENTES TEÓRICOS}

Desde el referente teórico, la investigación está soportada en la teoría normativa de los Stakeholders; Dovers y Handmer (1994) plantean que la responsabilidad social de las organizaciones ha venido transformándose desde, una responsabilidad social orientada únicamente a sus accionistas, luego trascendiendo de los accionistas y trabajadores, posteriormente ampliándose a los clientes, luego a la sociedad en general, luego al medio ambiente, hasta llegar al concepto actual de desarrollo sostenible; lo anterior partiendo del planteamiento de Dovers y Handmer (1994) en el que se afirma que las organizaciones inciden en el bien común de la sociedad y de los stakeholders que se afecten directamente por la actividad de la organización. (HANDMER, 2009)

De igual forma, Bleger (2004) plantea que en el contexto de las elecciones gubernamentales, el término stakeholders se refiere a cuerpos (organizados o no) de personas con un interés mutuo en relación con una organización de negocios, lo que lleva a concluir que el interés puede ser legalmente constituido dando apertura al contrato social (BLEGER, 2009), a su vez Agle, Mitchell y Sonnenfeld (1999) plantea la importancia de los valores empresariales como un todo; en el entorno empresarial se debe presentar como un complemento general, buscando alcanzar las metas financieras con los intereses de las directivas administrativas; Hillman y Keim (2001) en su teoría habla de cómo se pueden revertir las intenciones del grupo de inversores frente a la responsabilidad social de un grupo ajeno a la organización; de igual forma en su teoría Hendry (2001) hace referencia a los parámetros que deben constituir el stakeholders, trabajándolos desde un punto de vista ideal buscando la eficiencia de los stakeholders mediante un punto de engranaje que no sea ni muy alto ni muy bajo (HENDRY, 2009). Para Edward Freeman (1984) La teoría relaciona la complejidad de poder mantener los aspectos de interrelación entre todas las partes interesadas de una manera clara, precisa y sobre todo honesta. Sin importar la complejidad del contexto, se debe abarcar como un todo teniendo lo fácil de forma inmediata y lo difícil como algo que

\footnotetext{
${ }^{2}$ Lugar que es importante porque en él confluyen personas, vías de comunicación, etc., o porque en él se toman decisiones relevantes. Aspecto importante y delicado o difícil de abordar.
}

Revista ENIAC Pesquisa, Guarulhos (SP), V.6, n.2, jun.- dez. 2017 
tarda un poco más pero complementando las ideas se llega a conclusiones ideales dentro todos los grupos participantes de los stakeholders (FREEMAN, 2009).

En su planteamiento Jensen (2002) dice que se debe tener un solo concepto claro a la hora de formalizar las ideas dentro de los stakeholders ya que construir varias síntesis no permite generar el valor de las funciones objetivas. La focalización debe ser sólida, basada en una sola teoría que permita identificar claramente el enfoque que se requiere para su interpretación. (JENSEN, 2009). Por lo anterior, los recursos empleados por el sector público y privado se constituyen en un valor al cual todos los grupos de interés tienen acceso de forma directa, por la generación de informes financieros de las organizaciones y que definitivamente impactan en la confianza pública de estas corporaciones u organizaciones, generando un valor agregado a las mismas y que de una $u$ otra forma se constituyen en elementos que son directamente relacionados con los mecanismos de control que pueden ejercer los grupos de interés sobre las corporaciones y organizaciones objetos de estudio.

\section{METODOLOGÍA}

La investigación objeto de estudio es de corte descriptiva explicativa y sus asideros conceptuales se encuentran determinados en el estudio documental. Para conocer la causa de los incendios forestales en el Municipio de Ibagué, se revisó información documental, básicamente obtenida de la Corporación Autónoma Regional del Tolima - CORTOLIMA ${ }^{3}$, como autoridad ambiental en el Departamento del Tolima, el Ministerio del Medio Ambiente como entidad rectora de la gestión ambiental en Colombia (preside la Comisión Nacional Asesora para la Prevención y Mitigación de Incendios Forestales desde su creación en el año de 1993).

Los datos históricos de los incendios forestales se tomaron de un informe aportado por el área de Gestión del Riesgo de CORTOLIMA, un proyecto elaborado por la Subdirección de Planeación de CORTOLIMA y la información relacionada con datos climáticos fue suministrada por el IDEAM. Igualmente se consultó las páginas WEB de estas entidades y de otras, con el fin de revisar el grado de gestión que han desarrollado en el tema objeto del estudio.

\section{DISCUSIONES}

Teniendo como referencia que el total de las hectáreas afectadas por los incendios forestales en el Municipio de Ibagué fueron de 207.73/has, está en proceso de aprobación y ejecución un proyecto elaborado por la Autoridad Ambiental del departamento del Tolima, la CORPORACIÓN AUTÓNOMA REGIONAL DEL TOLIMA - CORTOLIMA y presentado a la cooperación internacional, donde se busca la "implementación de iniciativas para adelantar e iniciar procesos de restauración ecológica en áreas afectadas por

\footnotetext{
${ }^{3}$ Máxima Autoridad Ambiental en el Departamento del Tolima.
} 
incendios forestales" (CORTOLIMA, 2015), y se presenta un presupuesto de cuánto vale la restauración de dichas zonas.

\subsection{COSTOS GENERALES DE ESTABLECIMIENTO DE PLANTACIONES}

La tabla 1 permite evidenciar algunos de los recursos que son necesarios para la restauración, clasificados en los siguientes rubros como:

- $\quad$ Alquiler de vehículos para realizar las visitas, transportar materiales y personal.

- $\quad$ Equipos de última tecnología para realizar los estudios correspondientes.

- $\quad$ publicaciones como cartillas de educación ambiental.

- Asistencia técnica y administrativa con profesionales capacitados e idóneos

- Gastos administrativos como papelería.

Tabla 1 - Recursos necesarios para la restauración

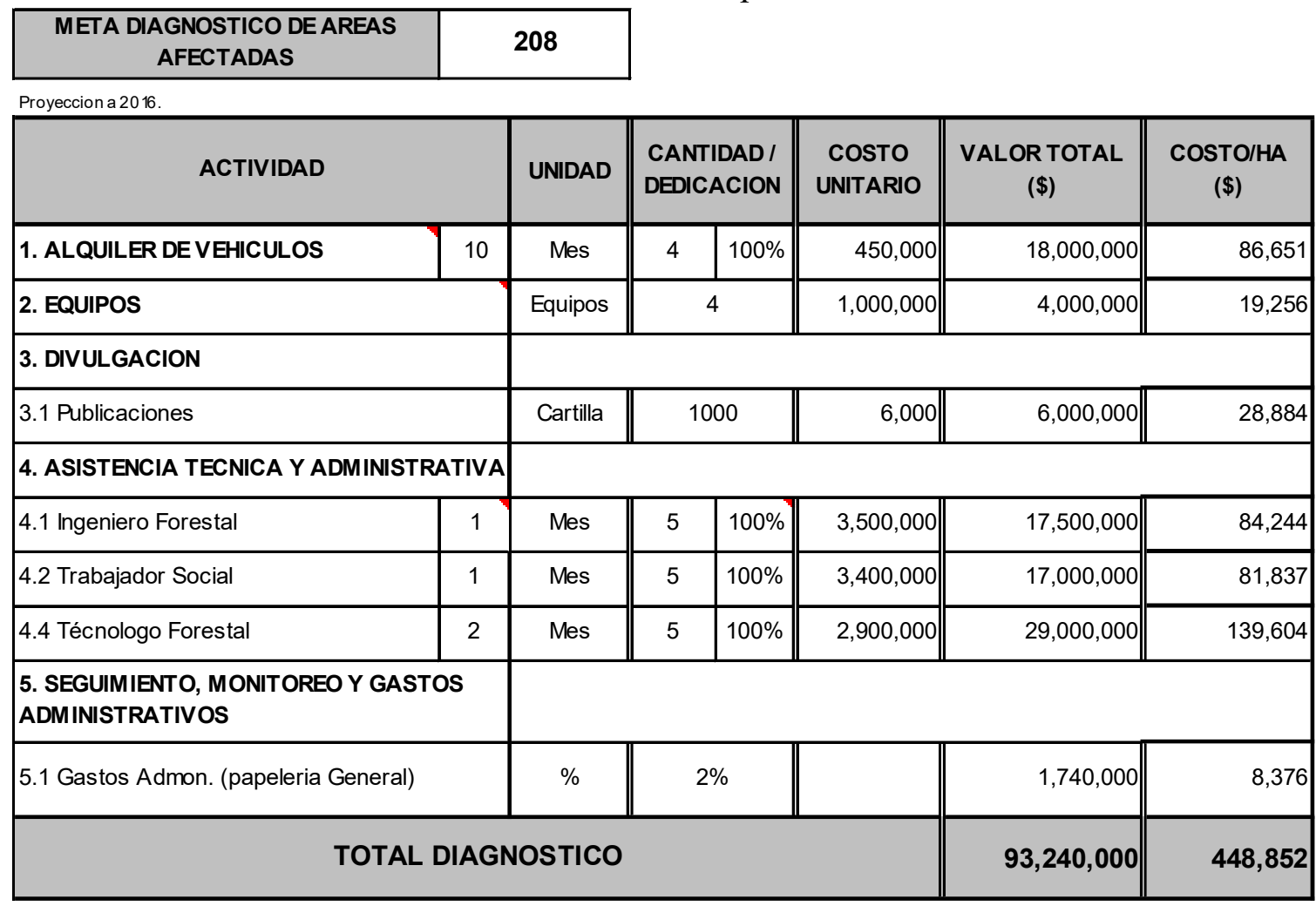

FUENTE: Construcción de los autores, área técnica subdirección de Planeación -

CORTOLIMA

Esto le cuesta a CORTOLIMA: CUATROCIENTOS CUARENTA Y OCHO MIL OCHOCIENTOS CINCUENTA Y DOS PESOS M/CTE (\$448.852.00) por cada

Revista ENIAC Pesquisa, Guarulhos (SP), V.6, n.2, jun.- dez. 2017 
hectárea, sin incluir los insumos básicos (plántulas, fertilizantes, herramientas, mano de obra). Esto quiere decir que, teniendo en cuenta solo estos ítem, la restauración de las 207.73/ha afectadas en el Municipio de Ibagué, le costaría a la entidad NOVENTA Y TRES MILLONES DOSCIENTOS CUARENTA MIL VEINTICINCO PESOS CON 96/100 M/CTE (\$93.240.025.96). Teniendo en cuenta que el alquiler de los vehículos y el pago de los funcionarios es mensual.

Tabla 2 - Gastos em alquiler de vehículo personal

\begin{tabular}{|c|c|c|c|c|c|c|}
\hline \multirow{3}{*}{\multicolumn{2}{|c|}{\begin{tabular}{|l} 
MUNICIPIO \\
VALOR POR HECTAREA \\
TOTAL HECTAREAS AFECT
\end{tabular}}} & \multicolumn{5}{|l|}{ IBAGUE - TOLIMA } \\
\hline & & & $\$ 448.852$ & & & \\
\hline & & TOTAL HECTAREAS AFECTADAS & \multicolumn{4}{|l|}{207,73} \\
\hline $\begin{array}{c}\text { MESES DEL } \\
\text { ANNO } 2015\end{array}$ & $\begin{array}{l}\text { CANTIDAD DE } \\
\text { INCENDIOS } \\
\text { FORESTALES }\end{array}$ & CAUSAS SOCIALES & $\begin{array}{c}\text { EFECTO } \\
\text { ECONOMICO }\end{array}$ & $\begin{array}{c}\text { EFECTO } \\
\text { AMBIENTAL }\end{array}$ & EFECTO ECOLOGICO & \begin{tabular}{|c|} 
PROPUESTA DE \\
ACCION POR PARTE \\
DEL LEGISLADOR \\
\end{tabular} \\
\hline ENERO & 29 & Descuido, Negligenciay altas temperaturas & \$ $\quad 1.184 .969,28$ & 264tha perdidas & Grandes perdida de vegetacion & En curso proyecto \\
\hline FEBRERO & 6 & Reactivacion de focos & $13.465,56$ & 0.03 ha perdidas & perdidas no tan signific ativas & En curso proyecto \\
\hline MARZO & 3 & Reactivacion de focos & $\$$ & 0 & no se reporto incendios & En curso proyecto \\
\hline ABRIL & 3 & intencional & $\$$ & 0 & no se reporto incendios & En curso proyecto \\
\hline MAYO & 26 & Descuido, Negligenciay altas temperaturas & $22,442,60$ & 0.05 ha perdidas & perdidas no tan significativas & En curso proyecto \\
\hline JUNIO & 29 & Descuido, Negligenciay altas temperaturas & $4.488,52$ & 0.01 ha perdidas & perdidas no tan signific ativas & En curso proyecto \\
\hline JUUO & 122 & Descuido, Negligenciay altas temperaturas & $\$ 20.938 .945,80$ & $46.65 /$ ha perdidas & Grandes perdida de vegetacion & En curso proyecto \\
\hline AGOSTO & 192 & Descuido, Negligenciay altas temperaturas & $\$ 53.992407,08$ & 120.29/ha perdidas & S Grandes perdida de vegetacion & En curso proyecto \\
\hline SEPTIEMBRE & 181 & Descuido, Negligenciay altas temperaturas & \$ $17.083 .307,12$ & 38.06 ha perdidas & Grandes perdida de vegetacion & En curso proyecto \\
\hline OCTUBRE & 0 & no hubo & $\$$ & 0 & no se reporto incendios & En curso proyecto \\
\hline NOVIEMBRE & 0 & no hubo & $\$$ & 0 & no se reporto incendios & En curso proyecto \\
\hline DICIEMBRE & 0 & no hubo & $\$$ & 0 & no se reporto incendios & En curso proyecto \\
\hline TOTAL & 591 & & $\$ 93.240,025,96$ & $207,73 \mathrm{HA}$ & & \\
\hline
\end{tabular}

FUENTE: Construcción de los autores, área técnica subdirección de Planeación -

CORTOLIMA

\subsection{DESCRIPCIÓN CAUSAS SOCIALES}

- Descuido, negligencia y altas temperaturas: representan un $15 \%-25 \%$ de los casos. En este apartado, las quemas agrícolas (en estos casos autorizados, pero en las que los autores perdieron el control del fuego extendiéndose éste por la superficie forestal colindante) están también entre las causas habituales. Otras causas son las colillas y hogueras mal apagadas, quema de basuras, trabajos forestales (Vélez Muñoz, R. , 2000).

- Intencional: Por falta de educación y conciencia ambiental existen personas que ocasionan incendios foréstales de forma intencional sin medir consecuencia alguna. Representan un 60-70 \% de los casos. Las motivaciones son variadas, siendo con diferencia las más comunes la quema no autorizada, ilegal e incontrolada de superficies agrícolas, ya sea para la eliminación de rastrojos o matorrales ("quema agrícola") o para la regeneración de pastos para el ganado. Otras motivaciones menos corrientes detrás de un incendio

Revista ENIAC Pesquisa, Guarulhos (SP), V.6, n.2, jun.- dez. 2017 
provocado son la piromanía, usos $\operatorname{cinegéticos}^{4}$, vandalismo, venganzas personales, especulación urbanística, bajar el precio de la madera, etc. Cabe señalar que el delito de incendio está tipificado en muchas legislaciones (Vélez Muñoz, R., 2000).

- Reactivación de focos: Es cuando se vuelve a generar el incendio.

\subsection{COSTOS BÁSICOS DE ESTABLECIMIENTO DE PLANTACIONES}

Para la restauración de estas hectáreas afectadas se tienen que asumir unos gastos básicos como insumos agrícolas, herramientas, elementos de protección personal. CORTOLIMA $^{5}$ hizo un estudio de mercado basado en tres cotizaciones diferentes para el año 2016, el cual se relaciona a continuación.

Tabla 4 - Custos básicos de estabelecimiento de plantaciones

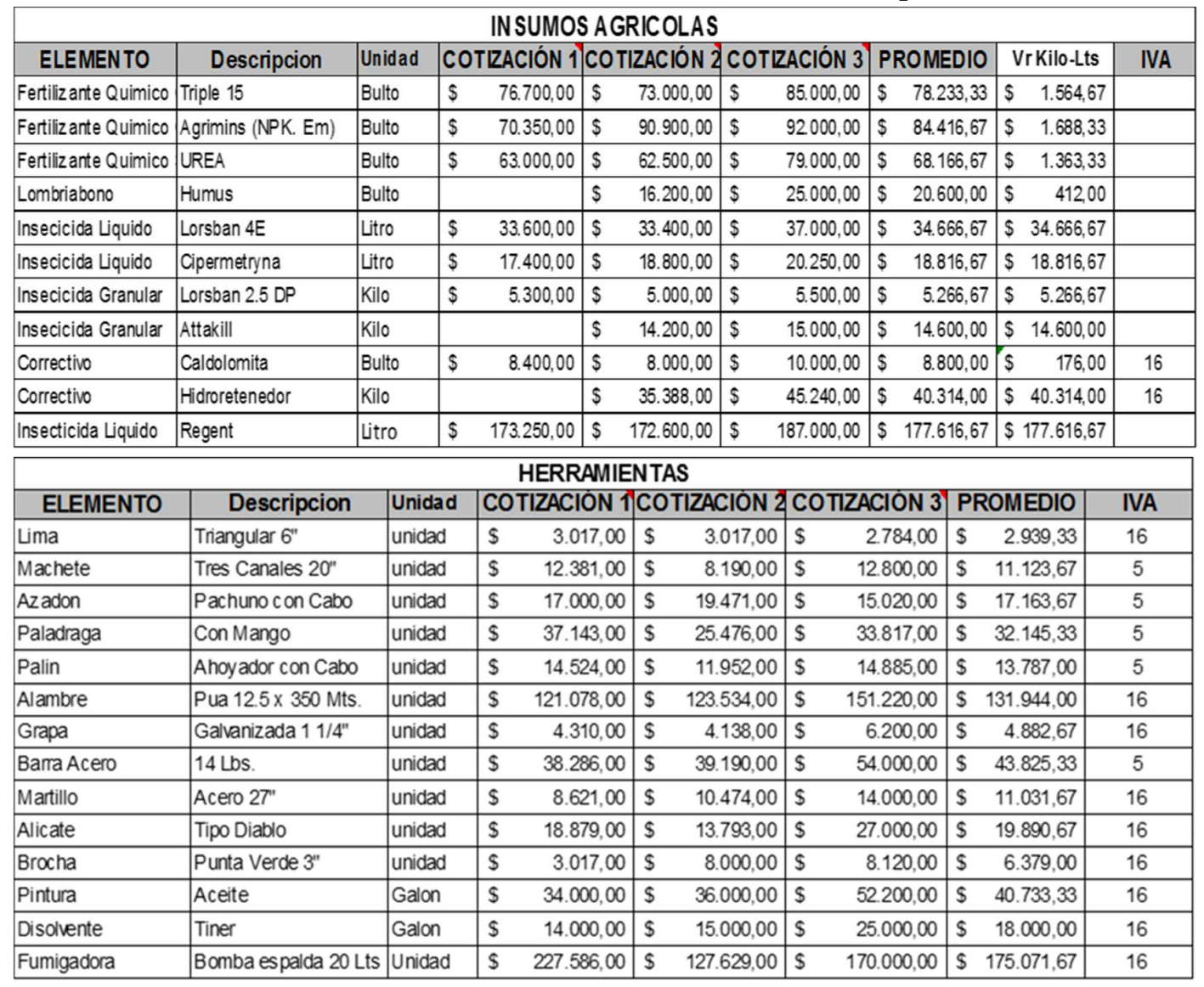

${ }^{4}$ la caza es la actividad o acción en la que se captura generalmente un animal.

${ }^{5}$ Máxima autoridad Ambiental en el Departamento del Tolima.

Revista ENIAC Pesquisa, Guarulhos (SP), V.6, n.2, jun.- dez. 2017 


\begin{tabular}{|c|c|c|c|c|c|c|c|c|c|c|}
\hline \multicolumn{11}{|c|}{ ELEMENTOS DE PROTECCIÓN PERSONAL } \\
\hline ELEMENTO & Descripcion & UNIDAD & $\mathrm{CO}^{-}$ & TIZACIÓN 1] & $\mathrm{COT}$ & TIZACIÓN 2 & $\mathrm{COT}$ & IZACIÓN 3 & & ROMEDIO \\
\hline Bota & Caucho Caña Alta & Par & $\$$ & $17.241,00$ & $\$$ & $18.966,00$ & $\$$ & $30.000,00$ & $\$$ & $22.069,00$ \\
\hline Guantes & Carnaza & Par & $\$$ & $4.310,00$ & $\$$ & $4.310,00$ & $\$$ & $7.000,00$ & $\$$ & $5.206,67$ \\
\hline Guantes & Nitrilo & Unidad & $\$$ & $4.310,00$ & $\$$ & $4.310,00$ & $\$$ & $7.000,00$ & $\$$ & $5.206,67$ \\
\hline Tatabocas & Desechable & Unidad & $\$$ & 603,00 & $\$$ & 603,00 & $\$$ & 603,00 & $\$$ & 603,00 \\
\hline Gafas & Seguridad & Unidad & $\$$ & $6.897,00$ & $\$$ & $6.897,00$ & $\$$ & $5.000,00$ & $\$$ & $6.264,67$ \\
\hline Sombrero & Tipo Pava & Unidad & $\$$ & $13.793,00$ & $\$$ & $12.931,00$ & $\$$ & $15.000,00$ & $\$$ & $13.908,00$ \\
\hline Camiseta & Manga Larga & Unidad & $\$$ & $14.655,00$ & $\$$ & $12.931,00$ & $\$$ & $10.000,00$ & $\$$ & $12.528,67$ \\
\hline Elemento & Descripcion & Unidad & $\mathrm{CO}^{\circ}$ & TIZACIÓN 1 & $\mathrm{CO}^{1}$ & TIZACIÓN 2 & $\mathrm{CO}^{\circ}$ & TIZACIÓN 3 & & ROMEDIO \\
\hline Plantula & $\begin{array}{c}\text { De } 25 \text { a } 50 \mathrm{Cm} \text { Altura } \\
\text { y Bolsa } 10^{*} 18 \text { Calibre } \\
1.5\end{array}$ & Plantula & $\$$ & $1.150,00$ & $\$$ & $1.160,00$ & $\$$ & 1. 180,00 & \$ & $1.163,33$ \\
\hline ELEMENTO & Descripcion & Unidad & $\mathrm{CO}^{\circ}$ & TIZACIÓN 1| & $\mathrm{CO}^{-1}$ & TIZACIÓN 2 & $\mathrm{CO}^{\circ}$ & IIZACIÓN 3 & & ROMEDIO \\
\hline Poste & $\begin{array}{l}\text { Plastico Cuadrado } \\
7 \times 7 \mathrm{Cm} \times 2.20 \text { Mts }\end{array}$ & Unidad & $\$$ & $23.850,00$ & $\$$ & $23.550,00$ & $\$$ & $24.200,00$ & $\$$ & $23.866,67$ \\
\hline Poste & $\begin{array}{l}\text { Madera } 10^{*} 10 \\
\text { Escuadria X2.20 Mts. }\end{array}$ & Unidad & $\$$ & $16.100,00$ & $\$$ & $16.950,00$ & $\$$ & $17.300,00$ & $\$$ & $16.783,33$ \\
\hline
\end{tabular}

FUENTE: Tablas suministradas por el área técnica subdirección de Planeación -

CORTOLIMA

En el siguiente cuadro nos muestra detalladamente la proyección de gastos básicos para la restauración de las 207.73 has perdidas en el Municipio de Ibagué, como son: la compra de insumos, pago de la mano de obra, herramientas, elementos de protección personal, transporte de insumos, el número de plántulas que se requieren sembrar por hectárea perdida, en este caso serían 1.110 la cual tiene un valor unitario de \$1.163.

Revista ENIAC Pesquisa, Guarulhos (SP), V.6, n.2, jun.- dez. 2017 
Tabla 5 - Muestra de la proyección de gastos básicos para la restauración

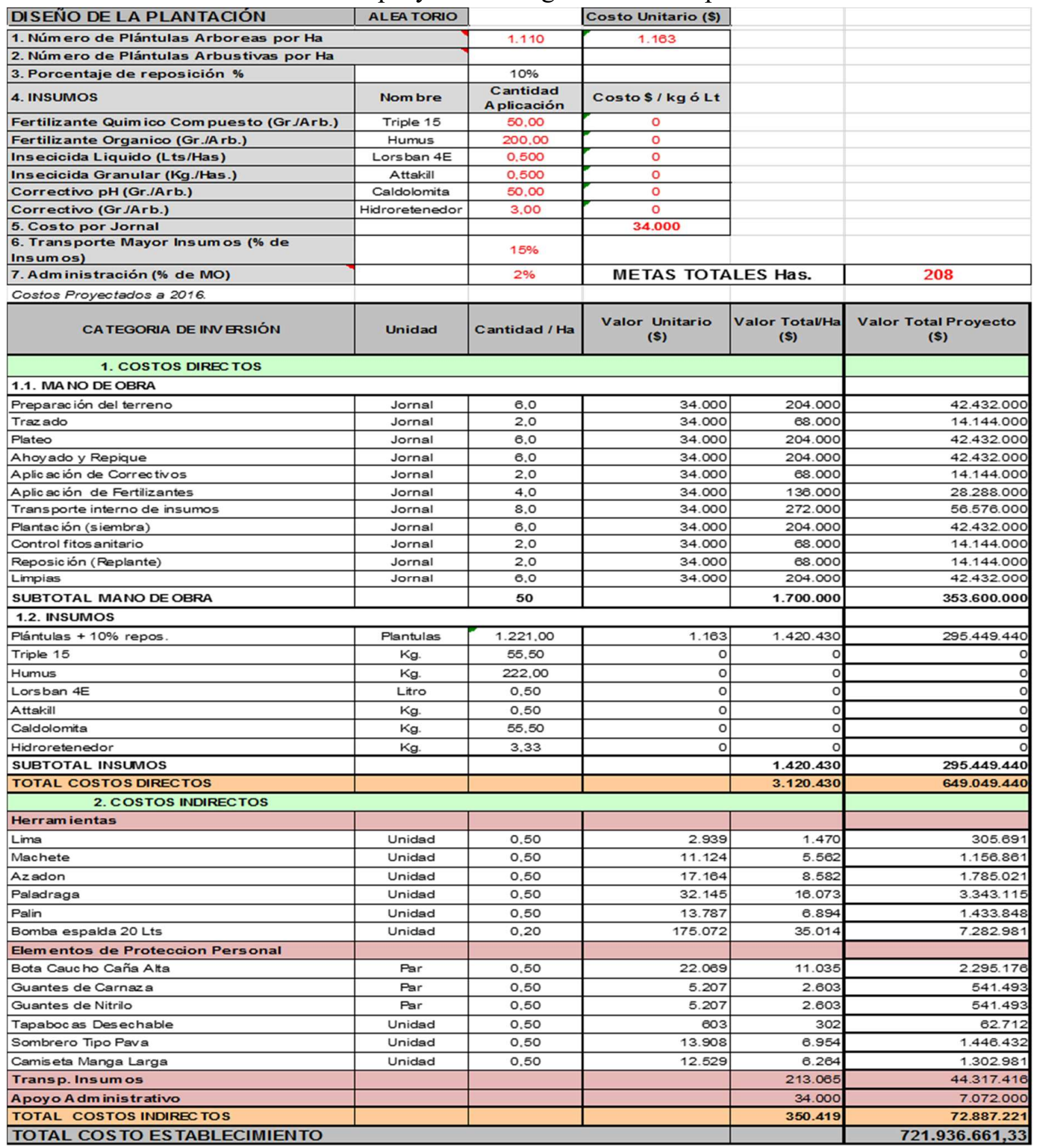

\section{CORTOLIMA}

FUENTE: Construcción de los autores, área técnica subdirección de Planeación -

El valor total de estos gastos básicos estipulados anteriormente en la proyección ascienden a la suma de SETECIENTOS VEINTIÚN MILLONES NOVECIENTOS TREINTA Y SEIS MIL SEISCIENTOS SESENTA Y UN PESOS CON 33/100 (\$721.936.661.33).

Revista ENIAC Pesquisa, Guarulhos (SP), V.6, n.2, jun.- dez. 2017 


\section{COSTOS TOTALES PARA EL ESTABLECIMIENTO DE PLANTACIONES}

Teniendo en cuenta la suma de los costos generales de establecimiento que ascienden A: NOVENTA Y TRES MILLONES DOSCIENTOS CUARENTA MIL VEINTICINCO PESOS CON 96/100 M/CTE (\$93.240.025.96), más los costos básicos de establecimiento de plantaciones que ascienden A: SETECIENTOS VEINTIUN MILLONES NOVECIENTOS TREINTA Y SEIS MIL SEISCIENTOS SESENTA Y UN PESOS CON 33/100 (\$721.936.661.33), al estado le costaría la restauración de las 207.73 has afectadas, un valor total de OCHOCIENTOS QUINCE MILLONES CIENTO SETENTA Y SEIS MIL SEISCIENTOS OCHENTA Y SIETE PESOS CON 29/100 (\$815.176.687.29).

\section{CONCLUSIONES}

Las causas por las cuales se presentaron los incendios forestales en el Municipio de Ibagué - Tolima, durante el año 2015, fueron presuntamente de origen antrópico (generados intencionalmente por algunas personas para la ampliación de la frontera agropecuaria, por negligencia, descuido o accidentes), reflejando la falta de conciencia ambiental en la población, como también de origen natural, principalmente por los efectos del cambio climático, lo que permite efectivamente proponer que se realicen procesos de trazabilidad respecto a los proyectos de educación ambiental que se realizan por parte de CORTOLIMA, puesto que sí se están haciendo las campañas de sensibilización, pero que al parecer por la información que se evidencia en la investigación, estas no han tenido el eco necesario en la población; de igual forma, Un apreciativo de cuánto le cuesta al estado la restauración de las zonas pérdidas, está proyectado en la suma de: OCHOCIENTOS QUINCE MILLONES CIENTO SETENTA Y SEIS MIL SEISCIENTOS OCHENTA Y SIETE PESOS COLOMBIANOS CON 29/100 (\$815.176.687.29), lo que se constituye en un valor bastante significativo y que se pudiera disminuir si se tuviera una adecuada participación en materia de conservación de la población.

Los incendios forestales dejaron grandes pérdidas, como fue la destrucción de la capa vegetal; generando, entre otras cosas la disminución en la producción de aguas para todos los usos; por esta causa se perdieron 207.73 hectáreas, debido a esto hubo desplazamiento de la fauna que habitaba en las zonas afectadas, rompiendo el ciclo de los ecosistemas, de igual forma, se evidencia claramente que el Municipio de Ibagué - Tolima, fue el más afectado por los incendios forestales en el Tolima, pues presentó un total de 591 incendios durante el recorrido del año 2015 a nivel del Departamento del Tolima, constituyéndose en uno de los desastres naturales apilados de mayor envergadura de los últimos 10 años y atentando contra la continuidad de los entes económicos que se surten del agua de estas quebradas.

- $\quad$ Se evidencia falta participación del sector privado en la prevención y mitigación de los incendios forestales, puesto que, según la investigación realizada, no se han encontrado 
empresas del sector privado que hayan o estén interesadas en formular proyectos para contribuir de alguna manera en la restauración de los daños dejados por los incendios forestales en el Municipio de Ibagué, permitiendo de esta forma que no se cumpla con las indicaciones establecidas por la alcaldía de Ibagué como fu hacer de Ibagué una ciudad sostenible.

\section{BIBLIOGRAFÍA}

Bleger, I. (2004). El enfoque de los stakeholders para la alta dirección. Recuperado el 17 de abril de 2008, Congreso de Adenag.

Contingencia, P. D. (s.f.).

Cortolima. (2 de Enero de 2012). Cortolima. Obtenido de:

http://www.cortolima.gov.co/cortolima/informe-pqr

Cortolima. (2015). Implementacion de iniciativas para adelantar e iniciar procesos de restauracion ecologica en areas afectadas por incendios forestales . Ibague: cortolima.

Dovers, S. R., Norton, T. W., \& Handmer, J. W. (1996). Uncertainty, ecology, sustainability and policy. Biodiversity \& Conservation, 5(10), 1143-1167.

Flórez-Guzmán, M. H., Hernández-Aros, L., \& Gallego-Cossio, L. C. (2016). Tableros de control como herramienta especializada: perspectiva desde la auditoría forense. Cuadernos de Contabilidad, 16(42).

Freeman, R. E. (1984). Stakeholder management: framework and philosophy. Pitman, Mansfield, MA.

Guzmán, M. H. F., \& Guerrero, C. A. P. (2015, July). El conocimiento estrategia gerencial de competitividad para las microempresas en colombia/managerial strategies to improve small business competitiveness. In Global Conference on Business \& Finance Proceedings (Vol. 10, No. 2, p. 1282). Institute for Business \& Finance Research.

Guzmán, M. H. F., Arbeláez, M. F. C., Balcázar, A. P. G., Hernández, E. D. A., Mejía, Y. T., \& González, L. D. T. (2015, July). Caracterizacion de las politicas ambientales implementadas por las empresas colombianas en concordancia con su objeto sociaL. In Global Conference on Business \& Finance Proceedings (Vol. 10, No. 2, p. 1287). Institute for Business \& Finance Research.

GUZMÁN, M. H. F., GIL, G. G., \& YATE, G. M. M. (2016). Caracterización de las políticas de responsabilidad social de los almacenes de grandes superficies-hipermercados-

Revista ENIAC Pesquisa, Guarulhos (SP), V.6, n.2, jun.- dez. 2017 
en colombia."un análisis desde las normas internacionales de auditoría nia. Pensamiento Republicano, (3).

Guzmán, M. K. F., \& Guzmán, L. H. F. (2015). La Responsabilidad Social Empresarial y el Marketing como Factores Generadoras de Valor en las organizaciones "Un análisis desde la teoría de los Stakeholders".

wnloads/teorias\%20de\%20stakeholders.pdf.

Handmer, J., \& Dovers, S. (2009). A typology of resilience: rethinking institutions for sustainable development. The Earthscan reader on adaptation to climate change.

Hassán, J. O. C., \& Guzmán, M. H. F. (2014, July). Incidencia de la política de sostenibilidad en el desempeño financiero de las empresas: analisis de un caso exitoso de sostenibilidad en colombia/incidence of sustainability policy in the financial performance of the companies: a research of a bought case to a successful case of sustainability in colombia. In Global Conference on Business \& Finance Proceedings (Vol. 9, No. 2, p. 1985). Institute for Business \& Finance Research.

Hendry, D. F., \& Doornik, J. A. (2001). PcGive 10: Empirical econometric modelling using. Timberlake Consultants.

Herrero Echavarría, J. A., Gómez, L. M., Díaz Quintero, G., Bravo, J. A., Alfaro, R. I., Battisti, A., ... \& Hennon, P. E. (2005). Criterios e indicadores de manejo forestal sostenible: una visión de futuro (No. 634.92097291 H565c). Ministerio de la Agricultura, La Habana (Cuba)..

Marcote, P. V., \& Suárez, P. A. (2005). Planteamiento de un marco teórico de la Educación Ambiental para un desarrollo sostenible. Revista electrónica de Enseñanza de las Ciencias, $4(1)$.

ONU, n. u. (01 de 01 de 2015). http://www.un.org/es/millenniumgoals/environ.shtml. Obtenido de http://www.un.org/es/millenniumgoals/environ.shtml.

Perdiguero, T. G., \& Reche, A. G. (Eds.). (2005). La responsabilidad social de las empresas y los nuevos desafios de la gestión empresarial (Vol. 52). Universitat de València.

Radio, R. L. (31 de 08 de 2015). RCN La Radio. Obtenido de:http://www.rcnradio.com/locales/el-tolima-pide-ayuda-por-incendios-forestales/

Santos Martínez, A., Hinojosa, S., \& Sierra Rozo, O. (2009). Proceso y avance hacia la sostenibilidad ambiental: la reserva de biosfera seaflower, en el caribe colombiano. Caribbean Notebooks; Vol. 13 (2009): Reserva de Biosfera Seaflower: Problemas Ambientales; 17 Revista Cuadernos del Caribe; Vol. 13 (2009): Reserva de Biosfera

Revista ENIAC Pesquisa, Guarulhos (SP), V.6, n.2, jun.- dez. 2017 
Seaflower: Problemas Ambientales; 17 Cahiers des Caraïbes; Vol. 13 (2009): Reserva de Biosfera.

Townsend, M. S., Aaron, G. J., Monsivais, P., Keim, N. L., \& Drewnowski, A. (2009).

Less-energy-dense diets of low-income women in California are associated with higher energy-adjusted diet costs. The American journal of clinical nutrition, 89(4), 1220-1226.

Vélez Muñoz, R. (2000). La defensa contra los incendios forestales: Fundamentos y experiencias (No. 634.9618 D313d). Madrid, ES: McGraw-Hill Interamericana.

Revista ENIAC Pesquisa, Guarulhos (SP), V.6, n.2, jun.- dez. 2017 